\title{
Autotraining using sustainable digital technologies: myth or reality
}

\author{
Margarita Filatova-Safronova ${ }^{1, *}$ and Daria Kuramshina ${ }^{1}$ \\ ${ }^{1}$ Kazan Innovative University named after V.G. Timiryasov (IEML), 42, Moskovskaya str., 420111, \\ Kazan, Russia,
}

\begin{abstract}
This study aimed to analyze the possibilities that modern digital technologies offer to the fields of health, psychotherapy, psychology and self-help. A review of various studies in the field of digital medicine with statistical data was conducted. The conclusions that researchers from different countries came to were similar: digital health applications are a practical solution that can be used to improve mental and psychological health. Moreover, digital psychotherapy can serve as a preventive tool to avert the development of mental disorders, increase the ability to deal with stress and mental problems, grow self-confidence, and more. The results of a study that was carried out using a Russian multimedia tool Master Kit are discussed. After using the program for three months, its users advanced in their ability to create an image of the desired outcome (a goal) and develop a plan to reach it. There is a noticeable difference in how goal-oriented the users became and their ability to independently self-train. Experimental data suggest that the tool can be effectively used to form and transform personal beliefs through a self-training format. More than half of the subjects talked about their satisfaction with the method, and more than one-quarter of them noted that their expectations from working with the program were fully satisfied.
\end{abstract}

\section{Introduction}

Global events that have changed the world over the last year have created a new normal. What we used to think of as a fantasy or a distant future turned out to be a reality. The issue of providing adequate psychotherapy to the general population has gained unprecedented urgency. The groups of people who needed psychotherapy the most were those infected with COVID-19 and their families, medical workers who worked in hospitals away from their loved ones, those who suffered from isolation and loneliness, and people who perceived the COVID-19 crisis as a potential threat to their well-being. Many people experienced negative thoughts and emotions, which triggered post-traumatic stress disorder even in people whom COVID-19 did not infect. The study [1] provides statistics that in China, $96.2 \%$ of residents infected with COVID-19 were diagnosed with post-traumatic stress disorder, $71.5 \%$ of medical workers suffered from an unstable emotional state, and $50.4 \%$ of doctors noted symptoms of depression.

\footnotetext{
*Corresponding author: 2592062@gmail.com
} 
Long-term social-distancing and self-isolation measures have made it more challenging to receive mental help and made it impossible to meet with therapists in a traditional inperson setting. There has been a significant increase in mental health professionals' workload in hospitals and a shortage of available specialists. During this period, online consultations, digital tools to get timely mental health, and digital psychotherapy rose in demand. Digital health solutions allow to avoid physical contact and make it possible to access electronic resources and online therapy at any time.

The psychotherapy and mental health industries have gradually moved to digital platforms. Accenture, a consulting company that studies trends in the use of various tech gadgets and artificial intelligence in healthcare, conducted an international online survey involving adults over 18 years old [2]. According to the survey, approximately half of the respondents used digital health applications (an increase by almost three times compared to 2014). The survey results in the U.S. showed that $81 \%$ of the patients ranked highly wearable digital health gadgets and $56 \%$ of the respondents noted the undeniable benefits of using artificial intelligence in surgery. In 2017, there were approximately 325,000 digital health applications available on Google Play and iTunes, the two most popular application platforms [cit. by 3].

Russian researchers A. Tsvetkova and A. Shishkin [4] used statistics from the consulting company Accenture and analytical company C.B. Insights and materials from a joint study by Econsultancy and Adobe. In their study, the authors provided a comparative assessment of the perception of various aspects of digital healthcare by the youth consumer segment and their degree of readiness to use digital health solutions. The study involved 380 Russians from 18 to 35 years old. It showed that the youth did not have a comprehensive understanding of all aspects of digital health. Most often, they used the Internet to look up information on hospitals and the services they offered, learn more about medicinal drugs (approximately $65 \%$ of the respondents), make a doctor's appointment online ( $48 \%$ of the respondents), or find information on various methods to treat a disease (40\%). Interestingly, the study also showed that modern youth did not plan on using any telehealth solutions in the future $(32 \%)$ and almost did not purchase electronic gadgets for health purposes $(9 \%)$. They also did not think of social media $(3 \%)$ or mobile applications $(2 \%)$ as reliable information sources. Meanwhile, the percentage of digital health users in the U.S. is much higher.

The use of digital technologies and mobile applications in healthcare has become more and more popular over the last few years. The analysis of recent studies demonstrates that researchers have made significant progress in the digital health industry. Virtual reality and artificial intelligence are successfully used in collaboration with gaming technologies, expanding psychotherapy opportunities. Digital therapy has seen positive results in treating depression, post-traumatic stress disorder, drug addiction, and more.

For example, Iyadurai L. et al. [5] conducted an online survey that involved 350 respondents to determine their readiness to receive new treatment methods after a traumatic event. The overwhelming majority of the respondents preferred to receive treatment via a mobile phone $(80.2 \%) .85 .7 \%$ of the respondents were willing to play Tetris, a computer game, to reduce intrusive memories for 10-20 minutes. Experimental data showed that new therapy methods reduce intrusive memories by $62 \%$ in a week than the control group. No side effects were observed.

Eshuis L.V et al. [6] raise the effectiveness of immersive treatment of post-traumatic stress disorder in their article. Their work provides an overview of the available data on the effectiveness of virtual reality exposure therapy (VRET) and augmented reality exposure therapy (ARET) as a treatment for post-traumatic stress disorder. VRET and ARET are relatively new digitally assisted psychotherapy methods that can improve post-traumatic stress disorder treatment by enhancing the patient's sense of presence during exposure therapy. Using ARET, digital feared stimuli are inserted into the physical space around the 
patient using the interactive digital device. With VRET, the user becomes even more immersed in the digital environment: it depicts feared stimuli using audio, video, and sometimes even physical objects or sensations. According to the authors, VRET is as effective as other psychotherapy methods and can act as an alternative to existing methods of treating post-traumatic stress disorder for patients who have not responded to previous treatment.

In their study, Garot $\mathrm{T}$. et al. [1] described an algorithm for working with digital applications for smartphones, tablets, and computers to collect longitudinal data to predict and track the dynamics of post-traumatic stress disorder. It could also be used to assess the relative effectiveness of methods aimed to prevent post-traumatic stress disorder immediately after the trauma and to inform the users about psychological signs that can appear during and after the traumatic event. If the application detects severe symptoms, it will ask the users to seek professional help from a psychotherapist. According to the authors of the study, working with this application is promising because such preventative measures can be accessible to many populations. The user will only need Internet access to work with the application, making it accessible from any digital device.

Renn B.N. et al. [7] conducted a study comparing patients' preferences suffering from depression for in-person or digital therapy methods. It showed that the main reason that influenced the decision to start psychotherapy to manage depression was where psychotherapy was available. Digital therapy has the advantage of no physical barriers. Out of 164 respondents, $44.5 \%$ preferred in-person psychotherapy and expressed concerns regarding the effectiveness of digital treatment, privacy risks, and the ability to achieve results independently. Simultaneously, $45.4 \%$ of the respondents chose digital therapy, with $25.6 \%$ choosing digital treatment with self-management and $19.7 \%$ preferring having an expert guide them through the digital treatment.

The aim of the study by Schuster R. et al. [8] was to demonstrate the effectiveness of combination treatment of depression. The experiment involved 174 adults who received conventional psychotherapy (in-person) and 166 adults who received extended combination therapy (including using the Internet). The authors concluded that the combination treatment led to a more significant reduction in the symptoms of depression, supported therapeutic progress, and improved mental health and quality of life. Simultaneously, the treatment's success did not appear to be connected with the combined in-person therapy's therapy direction.

Dr. Gnanavel S. discussed the issues regarding the usage of digital psychotherapy for children and teenagers. Vajawat B. et al. [10] provided an exciting overview of the use of digital gaming technologies in psychiatry. They demonstrated different applications of digital gaming technologies in psychotherapy for various cognitive learning and biofeedback disorders. For example, a survey showed that exergames, recreational games, and special cognitive educational games (such as Fitt and Hick, Big Brain Academy, Smart Aging, Wii, etc.) were used to prevent aging and dementia. A series of skill development and stimulus management mini-games (Guardian Angel, Clean House) can treat drug addiction and impulse control disorder. For treating depression, a wide range of CBT-based games ("SPARX") that have biofeedback at their foundation ("Freeze Farmer") were used. For biofeedback for Autism Spectrum Disorders, Exergames (Nintendo Wii and Mario and Sonics at the Olympics) and a serious game (Emotiplay). Games such as EndeavourRx, "Adventurous Dreamy High Flying Dragon," "Plan-it Commander," "A Journey of Wild Divinity," and others can be used to manage Attention Deficit Hyperactivity Disorder (ADHD).

The study considered the scope, application, and evidence of digital games in psychotherapy. Some of the main opportunities of applied video games in digital therapy were the following: the frequency of playing video games, involvement in order to complete 
digital exposure, accessibility of digital exposure, the ability to target a specific population group (the retirees), and the possibility of maintaining anonymity and confidentiality.

The review of studies carried out by Huckvale K. et al. [11] is of particular interest. It focused on the potential roles of mobile application in the treatment of mental illness. Given the great potential of using mobile applications as a safe and effective treatment for a range of mental illnesses, the authors drew attention to several factors that could not be ignored. These included the therapeutic approaches used (and how they complemented the treatment), the clinical safety of the application's content, data privacy and security, the likely constant interaction with the application, and the ways digital tools would integrate with the existing methods.

Bakker D. et al. $[12,13]$ provided a literature review focused on the research in mental health, preventative medicine, and mobile application design. The authors included a table that showcased the recommended features for iOS applications that were available. Based on the authors' analysis, there were sixteen such recommendations for MHapps. The study also provided an overview of mental health applications (MHapps), compared their effectiveness, and gave recommendations for creating better and more accurate applications. Three mobile applications were analyzed: mood tracking applications MoodKit, MoodPrism, and MoodMission. The sample consisted of 226 people randomly assigned into groups. The experiment results showed that using the applications led to an increase in mental well-being across all groups. The groups that used MoodKit and MoodMission experienced a decrease in depression and an increase in self-sufficiency. Using the applications as self-help and selfregulation tools was significantly more effective in the groups where individuals did not have a clinical diagnosis compared to the groups where individuals had clinically diagnosed mental disorders.

The authors concluded that these applications were a practical solution that could improve the users' mental health, increase confidence in their ability to cope with stress and mental problems, and serve as a tool to prevent mental disorders. However, the authors noted that the development of new MHapps would be primarily for research purposes and not to satisfy the end-users' needs.

The article by Ly K. et al. [14] described an experiment in which a mobile application that diagnosed the level of depression, anxiety, and stress in young people was used. The application simulated therapy by allowing the user to interact with a chatbot. Twenty-eight participants were randomly divided into two groups. One group (non-clinical) used the application and the automated chatbot Shim. The second group was a control group. The results demonstrated that the experimental group individuals showed a significant increase in mental well-being, stress management, and more. According to the experiment authors, the participants demonstrated high involvement compared to other studies on fully automated interventions, such as Woebot and Panoply.

The study results showed that monitoring their mental health allowed the subjects to increase their emotional self-control levels and their ability to recognize stressful situations and prepare for them in advance. Such self-help applications could be highly effective tools that supported many people's mental health. The authors suggested that monitoring one's mental state could be used as a preventative measure for the major depressive disorder because it allowed intervention while the depressive symptoms were still moderate.

Fitzpatrick K.K. et al. [15] studied the effectiveness of using a full automated chatbot (Woebot) in cognitive therapy for young people with the symptoms of depression and anxiety. The experiment involved 70 people aged 18-28 who were divided into two groups. One group used Woebot's chatbot to receive self-help content for two weeks (up to 20 sessions). The second group was the control group. The experimental group subjects interacted with Woebot's chatbot on an average of 12 times throughout the study. Statistical analysis of the data revealed a significant difference between the two groups. The individuals 
in the experimental group that interacted with the Woebot chatbot experienced a significant decrease in the symptoms of depression. Both groups experienced a decrease in anxiety. The authors concluded that chatbots were an attractive and effective way of participating in cognitive therapy.

Lui J. et al. [16] also reviewed studies on mobile applications to improve mental health. The authors analyzed over two hundred works in the mobile application field. They concluded that although almost all studies reported a significant decrease in negative symptoms resulting from using mobile applications, the studies lacked an empirical base. Moreover, some of the improvements that the subjects of certain studies experienced were not significantly different from the control group.

\section{Materials and methods}

Among Russian digital applications, the multimedia tool Master Kit deserves special attention [17]. It is an automated multimedia resource that allows the user to independently work with their limiting beliefs, fears, offenses, emotional situations in life, and self-esteem. The Master Kit method focuses on six fundamental beliefs: loving yourself unconditionally, my wishes, my emotions, my individuality, my life purpose, and desire for solitude.

Master Kit user talks through their individual and relevant limiting beliefs, repeating after an automated tool. The application broadcasts the text that the user has to repeat with an affirmative intonation and pauses. The user closes their eyes and repeats after the tool from 3 to 10 times, focusing on their feelings until they reach an appropriate positive emotional state.

The research we conducted in 2019 was aimed at finding out how the users' personal beliefs of the digital tool Master Kit were formed. The study involved 193 subjects: 96 users (experimental group, E.G.) who used the method at least five times a week and 97 users (control group, C.G.) who violated the Master Kit method's conditions. The trial was conducted over three months [18]. At the beginning of the trial and after its completion, data were obtained on the following:

- reflection, introspection, being conscious of the "here and now" state

- $\quad$ identification of individual goal-orientation

- $\quad$ creating an image of the result (the goal) and its outlines

- creating a plan to achieve a goal, identifying its positive aspects, possible difficulties, and risks

- $\quad$ self-training and self-suggestion, a transformation of personal beliefs.

As a diagnostic method for our trial, we used a survey of the users to work with each part of the Master Kit method and A. Ellis's method for diagnosing irrational beliefs.

\section{Results}

The study results showed that users' limiting beliefs had changed significantly after using Master Kit for three months. The analysis of the distribution of the respondents' answers on the development of their self-help skills according to the Pearson criterion $\chi^{2}$ showed that the answers did not significantly differ from each other. Users from both groups found it difficult to become conscious of their beliefs and goals and articulate them. They needed professional help to learn how to self-train correctly.

At the beginning of the trial, only $15 \%$ of the C.G. users and $11 \%$ of the E.G. subjects demonstrated that they were highly conscious of their "here and now" state and were able to reflect; $12 \%$ of the C.G. and $22 \%$ of E.G. demonstrated an above-average ability; $32 \%$ of the C.G. and $28 \%$ of the E.G. showed a medium level of ability; $33 \%$ of the C.G. and $30 \%$ of the 
E.G. showed a low level of reflection skills. $12 \%$ of both the C.G. and E.G. showed a high level of individual goal-orientation, $10 \%$ of C.G. and $22 \%$ of E.G. showed an above-average level of individual goal-orientation, $36 \%$ of C.G. and $43 \%$ of E.G. showed a medium level of individual goal-orientation, and $34 \%$ of C.G. and $20 \%$ showed a low level of individual goal-orientation. The statistics were similar for the users' ability to create an image of the result (the goal): $12 \%$ of C.G. and $11 \%$ of E.G. showed a high ability to create an image of their goal, $18 \%$ of the C.G. and $13 \%$ of the E.G. showed an above-average level of ability, $29 \%$ of the C.G. and $42 \%$ of the E.G. showed a medium level of ability, and $33 \%$ of the C.G. and $26 \%$ of the E.G. showed a low level of ability. When asked to create a plan to achieve the goal and identify its positive impact, only $7 \%$ of the C.G. and $10 \%$ of the E.G. showed a high level of ability to do so, $12 \%$ of the C.G. and $11 \%$ of the E.G. showed an above-average level of ability, $25 \%$ of the C.G. and $28 \%$ of the E.G. showed a medium level, and $48 \%$ of the C.G. and $43 \%$ of the E.G. showed a low level of ability to create a plan to achieve the goal and identify its positive aspects, possible difficulties, and risks. During the initial stage of the trial, the users also demonstrated a low level of self-training skills: only $6 \%$ of C.G. and $7 \%$ of E.G. showed a high skill level, $14 \%$ of C.G. and $11 \%$ of E.G. showed an aboveaverage skill level, $26 \%$ of the C.G. and $28 \%$ of the E.G. showed a medium skill level, and $48 \%$ of C.G. and $46 \%$ of E.G. showed a low ability to independently self-train and transform social and personal beliefs in order to reach their goals.

It is fair to say that individuals in both groups needed help learning how to analyze their mental state, correctly articulate their goals, become aware of their goals, and make a plan to reach them. A large number of the individuals needed professional help to properly self-train. Figure 1 provides the experimental group users' data at the beginning of the trial and three months after for five different scales.

The experimental group experienced significant changes in their skills after three months of working with Master Kit. Notable changes in the results of the two groups according to the $\chi^{2}$ criterion was observed in the skills of creating an image of the result (the goal) and developing a plan to achieve the goal $(\mathrm{p} \leq 0.05)$ as well as in the level of individual goalorientation and the ability to independently self-train $(\mathrm{p} \leq 0.01)$.

Three months after the start of the trial, $16 \%$ of the C.G. and $21 \%$ of the E.G. showed a high level of the ability to reflect, $15 \%$ of the C.G. and $26 \%$ of the E.G. showed an aboveaverage level of ability, $31 \%$ of the C.G. and $30 \%$ of the E.G. showed a medium level of ability, and $30 \%$ of C.G. and $15 \%$ of E.G. showed a low level of ability to reflect and become conscious of their mental state. A significant difference between the E.G. and C.G. was observed regarding the individual goal-orientation: $12 \%$ of the C.G. and $22 \%$ of the E.G. had a high level, $16 \%$ of the C.G. and $30 \%$ of the E.G. showed an above-average level, $25 \%$ of the C.G. and $32 \%$ of the C.G. showed a medium level, and $39 \%$ of the C.G. and $8 \%$ of the E.G. showed a low level of individual goal-orientation. There were no significant changes in the ability to create an image of the result (the goal) while using the tool. $17 \%$ of the C.G. and $23 \%$ of the E.G. showed a high level of ability, $17 \%$ of the C.G. and $30 \%$ of the E.G. showed an above-average level of ability, $29 \%$ of the C.G. and $27 \%$ of the E.G. had a medium level of ability, and $29 \%$ of the C.G. and $12 \%$ of the E.G. remained with the low level of ability to outline a goal. The ability to create a plan to achieve a goal changed: $8 \%$ of the C.G. and $22 \%$ of the E.G. showed a high level of ability, $13 \%$ of the C.G. and $20 \%$ of the E.G. showed an above-average level of ability, $24 \%$ of the C.G. and $28 \%$ of the E.G. showed a medium level of ability, and $47 \%$ of the C.G. and $22 \%$ of the E.G. showed a low level of ability. $8 \%$ of the C.G. and $18 \%$ of the E.G. showed a high level of self-training skills, $13 \%$ of the C.G. and $23 \%$ of the E.G. showed above-average skills, $25 \%$ of the C.G. and $24 \%$ of the E.G. had medium skills, and $46 \%$ of the C.G. and $27 \%$ of the E.G. still had low skills. 


\section{1. reflection, being conscious of the "here and now" state}

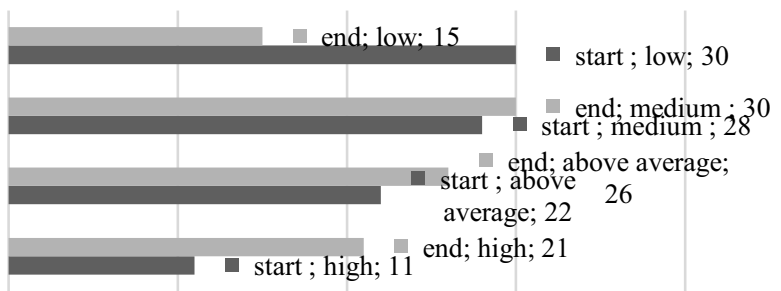

and $\mathbf{m}$ start

\section{2. individual goal-orientation}

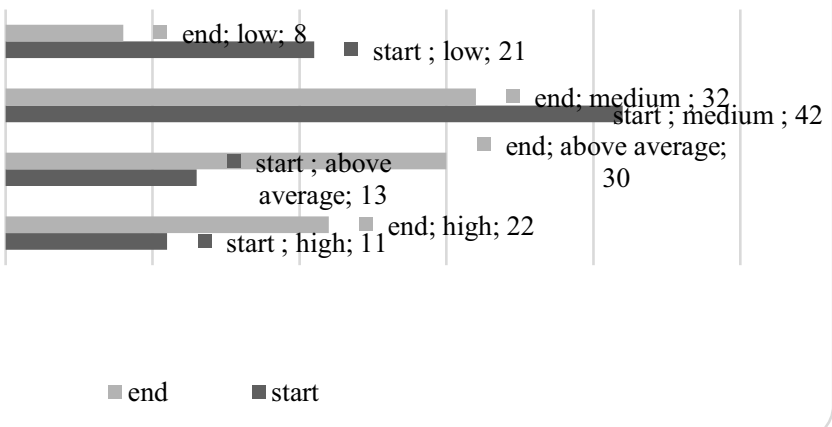

\section{3. creating an image of the result (the goal)}

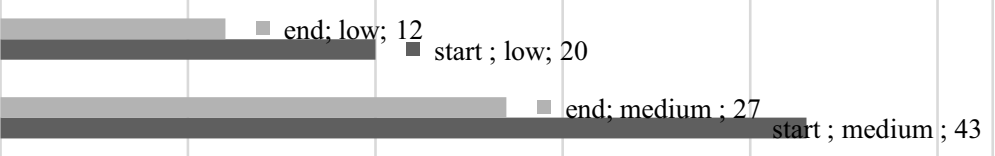
start ; above end; above average; average; 22

start ; high; 12

end; high; 23

mend $\quad$ start 


\section{4. creating a plan to achieve the goal}

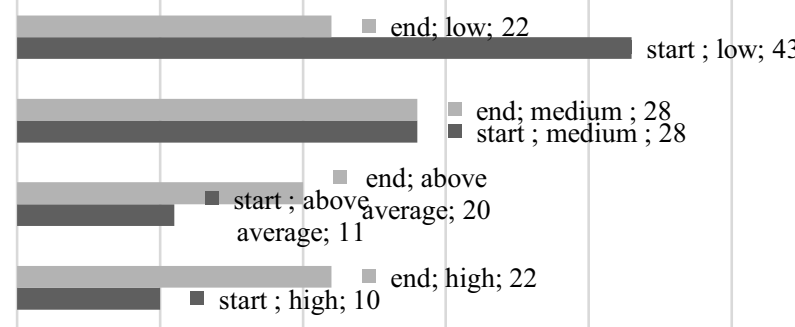

and start

\section{5. self-training and self-suggestion}

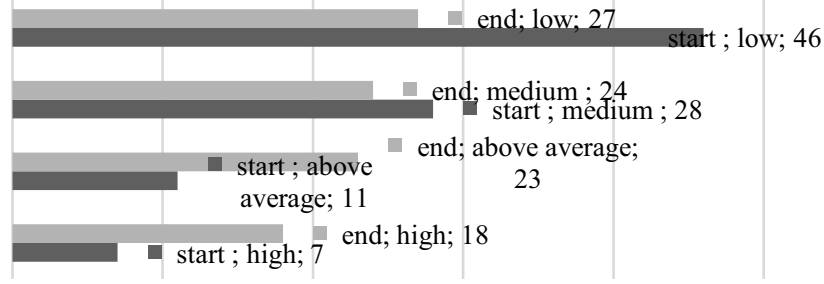

$\square$ end $\quad$ start

Fig. 1. Skill distributions in the experimental group.

A satisfaction survey showed that the results and first impressions almost matched their expectations for almost half of the respondents. $28 \%$ of the respondents noted that their expectations were fully met. A fifth of the respondents improved their relationships $(20.9 \%)$. $17.6 \%$ of the respondents improved either their health or the health of their loved ones. The most significant changes the respondents experienced were in their finances, with $27.5 \%$ claiming that they improved them because of Master Kit. 14.3\% of the respondents found their life purpose, $36 \%$ noted that the method helped them to identify and remove mental blocks and limiting beliefs that interfered with their lives, $31.5 \%$ learned to perceive life events through a positive lens, $18.9 \%$ learned how to set the right goals and develop direct plans to reach them, and $8.1 \%$ became more disciplined.

Overall, the experimental group users who regularly practiced the method mastered it, were generally satisfied with it, and thought of the changes in their lives as significant. These results most likely led to a transformation of their goal-setting beliefs.

Table 1 provides the average values of beliefs in the E.G. at the beginning and the end of the trial. At the beginning of the trial, Master Kit users did not show deviations from the average values: the subjects were characterized by irrational beliefs, such as the tendency to catastrophize, doing things out of "musterbation" (unrealistic high expectations for themselves or others), and low frustration tolerance. To understand their goals, desires, and aspirations, Master Kit users changed their goal-setting beliefs. Daily practice with the 
Master Kit method showed that the users experienced a new understanding of their life purpose. However, the digital tool could not remove the subjects' irrational beliefs. The difference between the results at the beginning of the trial and the end is not significant.

Table 1. Average values in the experimental group.

\begin{tabular}{|l|c|c|}
\hline \multicolumn{1}{|c|}{ Beliefs / trial stages } & beginning & end \\
\hline Tendency to catastrophize & 21.35 & 20.34 \\
\hline $\begin{array}{l}\text { Doing things out of "musterbation" (high expectations for } \\
\text { themselves) }\end{array}$ & 19.69 & 18.04 \\
\hline $\begin{array}{l}\text { Doing things out of "musterbation" (high expectations for } \\
\text { others) }\end{array}$ & 21.82 & 19.65 \\
\hline Self-esteem and ability to think rationally & 22.64 & 21.3 \\
\hline Frustration tolerance & 21.91 & 22.6 \\
\hline
\end{tabular}

\section{Discussion}

Our data showed that the users of the multimedia tool Master Kit ranked it highly and noted the apparent advantages of independently using a digital platform to work on their limiting beliefs, emotional states, self-esteem, and more. Our research aimed to understand how, when, and for how long this application could be used in clinical practice, self-therapy, and other areas.

We believe that a three-month period is sufficient to start managing relevant negative mental states, such as resentment, fears, and feelings of loneliness. This time frame allows mastering the self-training and self-suggestion techniques. Therefore, we can conclude that digital psychotherapy is effective after short-term use. However, a transformation of the existing limiting beliefs and a formation of new positive ones require a longer time. Limiting beliefs such as "my individuality" and "my life purpose" require digital support for a much more extended period of time.

There were professional and experienced individuals who continued to carry out daily tasks using the tool among the users of the Master Kit method. We continued to work with them to research the impact of digital therapy on the users' personal traits, such as conscious life orientation, intuition depth, anticipatory consistency, and more.

We believe that the results demonstrate Master Kit's effectiveness. It can be recommended to training centers that use self-training activities to transform goal-setting abilities, psycho-consultants, and other interested professionals. This method can be applied to many different social and age groups.

\section{Conclusions}

Psychotherapists' attitude to virtual reality is more positive than negative. Self-training activities of the Master Kit users can be considered adequate. Daily tasks lead to a transformation of personal limiting beliefs and allow the users to become more conscious of their life purpose.

Transformation of limiting beliefs is a gradual process that includes the following stages: reflective, goal-orientation, goal visualization, identification of a plan to achieve the goal and possible risks, and self-regulation.

The users of the Master Kit method at first need external help to articulate their life goals. It is difficult for them to independently set the right goal and create a plan to achieve it. The trial subjects who used the Master Kit method regularly mastered the self-training technique, we are satisfied with the method, and highly ranked the changes in their lives. These users had a significant improvement in their ability to visualize their goals and determine how to 
reach them. After only three months of working with this digital tool, almost half of the users were satisfied with the results in their personal lives.

It is common for those who are starting to work with Master Kit to have irrational limiting beliefs that can cause stress. The new users are characterized by a tendency to catastrophize, doing things out of "musterbation" (unrealistic high expectations for themselves or others), low frustration tolerance, and anticipatory failure.

The obtained experimental data support the initial theory that Master Kit can form and transform personal beliefs through auto-training.

Acknowledgements

The article was executed with the support of the Public Competition for grant and scholarship support for the publication activity of Kazan Innovative University named after V.G. Timiryasov (IEML)

\section{References}

1. Gargot, T., Kisand, H., Miguel, A., Tanyeri, S., Soron, T.R., Serene, B., Feron, G., Žaja, N., Valdés-Florido, M.J, Soto-Angona, O., Frankova, I.: Preventing Post Traumatic Stress Disorder in the general population induced by trauma during the COVID pandemic: A simple brief intervention based on cognitive science that could be delivered digitally. European Journal of Trauma \& Dissociation. Vol. 5 (2), (2021).

2. 2018 consumer survey on digital health. Accenture Consulting (2018), https://www.accenture.com/ t20180306T103559Z_w_/us-en/_acnmedia/ PDF71/accenture-health-2018-consumer-surveydigital-health.pdf last accessed 2021/02/15, last accessed 2021/02/02.

3. McKay, F., Slykerman, S., Dunn, M.: The App Behavior Change Scale: Creation of a Scale to Assess the Potential of Apps to Promote Behavior Change. JMIR Mhealth Uhealth. Vol.7 (1), (2019).

4. Tsvetkova, A.B., Shishkin, A.V.: Evaluation of the digital medicine perception by the youth consumer segment. Statistics and Economics. Vol. 15 (6), 46-57 (2018).

5. Iyadurai, L., Blackwell, S.E., Meiser-Stedman, R., Watson, P.C., Bonsall, M.B., Geddes, J.R., Nobre, A.C., Holmes, E.A.: Preventing intrusive memories after trauma via a brief intervention involving Tetris computer game play in the emergency department: A proof-of-concept randomized controlled trial. Molecular Psychiatry. Vol. 23 (3), 674-682 (2018).

6. Eshuis, L.V., Gelderen, M.J., Zuiden, M., Nijdam, M.J., Vermetten, E., Olff, M., Bakker, A.: Efficacy of immersive PTSD treatments: A systematic review of virtual and augmented reality exposure therapy and a meta-analysis of virtual reality exposure therapy. Journal of Psychiatric Research (2020), https://www.sciencedirect.com/science/article/pii/S002239562031089X, last accessed 2021/02/003.

7. Renn, B.N., Hoeft, T.J., Lee, H.S., Bauer, A.M., Areán, P.A.: Preference for in-person psychotherapy versus digital psychotherapy options for depression: survey of adults in the U.S. Digital Medicine. Vol. 6, (2019).

8. Schuster, R., Laireiter, A.R., Berger, T., Moritz, S., Meyer, B., Hohagen, F., Klein, J.P.: Immediate and long-term effectiveness of adding an Internet intervention for depression to routine outpatient psychotherapy: Subgroup analysis of the EVIDENT trial. Journal of Affective Disorders, Vol. 274, 643-651 (2020).

9. Gnanavel, S.: Computerised psychotherapy for children and adolescents: Opportunities and challenges. Asian Journal of Psychiatry. Vol. 24, 118-119 (2016). 
10. Vajawat, B., Varshney, P., Banerjee, D.: Digital Gaming Interventions in Psychiatry: Evidence, Applications and Challenges. Psychiatry Research. Vol. 295, (2021).

11. Huckvale, K., Nicholas, J., Torous, J., Larsen, M.E.: Smartphone apps for the treatment of mental health conditions: status and considerations. Current Opinion in Psychology. Vol. 36, 65-70 (2020).

12. Bakker, D., Kazantzis, N., Rickwood, D., Rickard, N.: Mental Health Smartphone Apps: Review and Evidence-Based Recommendations for Future Developments. JMIR Mental Health. Vol. 3(1), (2016).

13. Bakker, D.: A randomized controlled trial of three smartphone apps for enhancing public mental health. Behaviour Research and Therapy. Vol. 109, 75-83 (2018).

14. Ly, K.H., Trüschel, A., Jarl, L.: Behavioural activation versus mindfulness-based guided self help treatment administered through a smartphone application: a randomised controlled trial. BMJ Open. Vol. 4 (1), (2014).

15. Fitzpatrick, K.K., Darcy, A., Viefhile, M.: Delivering Cognitive Therapy to Young Adults with symptoms of depression and anxiety using a fully automated conversational agent (Woebot): a randomized controlled trial. JMIR Ment Health. Vol. 4 (2), (2017).

16. Lui, J., Marcus, D.K., Barry, C.T.: Evidence-Based Apps? A Review of Mental Health Mobile Applications in a Psychotherapy Context. Professional Psychology: Research and Practice. Advance online publication. (2017).

17. Master Kit, https://super-ego.info/master-kit/, last accessed 2021/02/15.

18. Filatova-Safronova M.A., Kuramshina D.R.: Semantic designs for the purpose of university graduates during autotraining activities: Education and law. Vol. 9. p 350-357 (2020). 